\title{
MUTANTS WITH HETERORESISTANCE TO AMPHOTERICIN B AND FLUCONAZOLE IN CANDIDA
}

\author{
Claudino, A.L.R. ${ }^{1}$; Peixoto Junior, R.F. ${ }^{1}$ Melhem, M.S.C. ${ }^{2}$; Szeszs, M.W. ${ }^{2}$; Lyon, J.P. ${ }^{3 *}$; Chavasco, J.K. ${ }^{1}$; Franco, M.C. ${ }^{1}$ \\ ${ }^{1}$ Departamento de Ciências Biológicas, Universidade Federal de Alfenas, Alfenas, MG, Brasil; ${ }^{2}$ Instituto Adolfo Lutz, São \\ Paulo, SP, Brasil; ${ }^{3}$ Instituto de Pesquisa e Desenvolvimento da Universidade do Vale do Paraíba, São José dos Campos, SP, \\ Brasil.
}

Submitted: May 16, 2008; Returned to authors for corrections: January 09, 2009; Approved: June 28, 2009.

\begin{abstract}
Several studies have reported the occurrence of infections caused by Candida yeasts as well as the increasing prevalence of non albicans species. The aim of the present work is focused on the obtaining of heteroresistance to amphotericin B and fluconazole in Candida species using two distinct methodologies: selection and induction. Resistant samples were obtained by selective pressure using a medium with fluconazole for growth, followed by growth in a medium with amphotericin B. The selective pressure was also created beginning with growth in amphotericin B medium followed by growth in fluconazole medium. Concomitantly, samples were submitted to the induction of resistance through cultivation in increasing concentrations of fluconazole, followed by cultivation in increasing concentrations of amphotericin B. Subsequently, the induction began with amphotericin B followed by fluconazole. Three samples resistant to fluconazole and amphotericin B were obtained, two by induction (C. glabrata and $C$. tropicalis) and one by selection (C. tropicalis). Both C. tropicalis originated from the same wild sample. After successive transfers for drug free medium, only the sample obtained by selection was able to maintain the resistance phenotype. These results suggest that the phenotype of heteroresitance to fluconazole and amphotericin B can be produced by two methodologies: selection and induction.
\end{abstract}

Key words: Amphotericin B, antifungal agents, Candida spp., fluconazole, heteroresistance, resistant mutants.

\section{INTRODUTION}

Candida yeasts are opportunistic pathogens that have been widely associated to severe infections in immunosuppressed individuals. The most frequently isolated species is C. albicans, but other Candida species have emerged as important pathogens (22). The use of antifungal agents, especially fluconazole, might lead to the selection of intrinsically resistant species such as $C$. krusei, or species as C. glabrata, in which the resistance is easily induced.

Heteroresistance is the expression of different resistance profiles in subpopulations of a strain (18). Mondon et al. (15)

*Corresponding Author. Mailing address: Instituto de Pesquisa e Desenvolvimento da Universidade do Vale do Paraíba, São José dos Campos-SP, Brasil. Av. Shishima Hifumi, 2911 Zip Code: 12244-000 - Bairro Urbanova , São José dos Campos - SP Phone (12) 3947-1000.; E-mail: julianalyon@yahoo.com.br 
investigated serial isolates of Cryptococcus neoformans obtained from two infected patients, demonstrating that each isolate produced cultures with heterogeneous compositions of susceptibility to fluconazole. Besides that, these authors verified that the proportion of subpopulations resistant to fluconazole increased steadily over time and that this selection process was reproduced in vitro. In the literature, studies focused on experimental induction of resistance have demonstrated that the development of in vitro resistance to fluconazole in $C$. tropicalis could be correlated with an experimental model of disseminated candidiasis (1). Similarly, a previous report performed by Bouchara et al (4) provided evidence that the selection of azole-resistant mutants of C. glabrata may occur in vivo after fluconazole administration, which would explain, therefore, clinical failure of antifungal therapy.

In the present article, we employed two in vitro methods, induction and selection, in order to obtain fluconazole and amphotericin B resistant mutants. This approach may provide additional information to improve the comprehension of the mechanisms involved in the resistance of Candida species.

\section{MATERIALS AND METHODS}

Samples: Thirty clinical isolates belonging to Adolfo Lutz institute, São Paulo, Brazil, were used in this study. The isolates were obtained from HIV+ and HIV- patients with erythematous oral candidiasis and comprised 22 C. albicans, 3 C. tropicalis, 3 C. parapsilosis and 2 C. glabrata. The reference samples C. parapsilosis ATCC 22019 and C. krusei ATCC 6258 were used as quality control organisms, being included each time a set of isolates was tested (16). All isolates were subcultured $24 \mathrm{~h}$ prior to testing.

Susceptibility testing: The antifungal susceptibility to fluconazole and amphotericin B was initially verified by Etest (AB BIODISK, Solna, Sweden). This test was performed on Mueller-Hinton agar plates supplemented with 2\% glucose and methylene blue $(0.5 \mu \mathrm{g} / \mathrm{ml})$ (Hardy
Diagnostics, Santa Maria, CA) according to the CLSI M44-A disc-diffusion method (21). Susceptibility to amphotericin B and fluconazole was also evaluated by the microdilution method as recommended by the Clinical Laboratory Standards Institute, [CLSI document M27A]. Stock solutions of amphotericin B (1600 $\mu \mathrm{g} / \mathrm{ml}$; Sigma, St. Louis, MO, USA) and fluconazole (5120 $\mu \mathrm{g} / \mathrm{ml}$; Pfizer, São Paulo Brazil) were prepared in dimethylsulphoxide and water, respectively.

Selection of heteroresistant isolates: This experiment was performed in agreement with the methodology proposed by $\mathrm{Xu}$ et al. (29) with modifications. The ability to grow in the presence of antifungal agents was evaluated in two stages: screening and selection of phenotypes resistant to the drug.

1) Screening: After cultivation for $24 \mathrm{~h}$ at $35^{\circ} \mathrm{C}$ in Sabouraud's broth, the 30 Candida spp. samples were inoculated in two tubes containing saline solution. The first tube was prepared with a final concentration of $10^{4} \mathrm{cfu} / \mathrm{ml}$ and the second one with final concentration of $10^{7} \mathrm{cfu} / \mathrm{ml}$. Those suspensions were inoculated in potato agar with fluconazole $(8 \mu \mathrm{g} / \mathrm{ml})$ and RPMI agar with addition of amphotericin B $(1.0 \mu \mathrm{g} / \mathrm{ml})$. The plates were incubated for $24 \mathrm{~h}$ at $35^{\circ} \mathrm{C}$. The choice of the antifungal concentrations was made according to the endpoint values established by Cuenca-Estrella et al. (6).

2) Selection: Selection $A(\mathrm{Fz} / \mathrm{AB})$ : The isolates that were not able to grow in the presence of fluconazole on the screening stage were inoculated in $3 \mathrm{ml}$ of Yeast Extract Peptone Dextrose broth (YEPD) (Difco, EUA) and incubated at $35^{\circ}$ C. After $24 \mathrm{~h}$, the tubes were centrifuged $(4000 \mathrm{rpm}$ for 5 min) and washed with saline solution. Cells were suspended in saline solution in a concentration of $10^{9} \mathrm{cfu} / \mathrm{ml}$. This suspension was inoculated in potato agar with fluconazole $(8 \mu \mathrm{g} / \mathrm{mL})$ and incubated at $35^{\circ} \mathrm{C}$ for $48 \mathrm{~h}$. The colonies with visible growth were selected randomly and cultured in $3 \mathrm{ml}$ of YEPD broth at $35^{\circ} \mathrm{C}$. After $24 \mathrm{~h}$, tubes were centrifuged (4.000 rpm for $5 \mathrm{~min}$ ) and washed with saline solution. A 
suspension of $10^{9} \mathrm{cfu} / \mathrm{ml}$ was prepared and inoculated in RPMI agar with amphotericin B $(1.0 \mu \mathrm{g} / \mathrm{ml})$. After incubation at $35^{\circ} \mathrm{C}$ for $48 \mathrm{~h}$, the colonies that demonstrated visible growth were randomly selected and cultured in Sabouraud agar at $37^{\circ} \mathrm{C}$ for $24 \mathrm{~h}$ for MIC determination. Selection B $(\mathrm{AB} / \mathrm{Fz})$ : This procedure was developed exactly as selection A, but the selected samples were those that did not present growth in the presence of amphotericin B on the screening stage and the order of the antifungals was inverted, initiating with amphotericin B. The reference samples $C$. parapsilosis (ATCC 22019) C. krusei (ATCC 6258), were applied in all tests as susceptibility and resistance controls, respectively. Isolates were classified as possibly heteroresistant when colonies grew on a plate containing amphotericin B or fluconazole.

Induction of heteroresistant isolates: Induction $A(\mathrm{Fz} /$ $A B)$ : After cultivation in agar Sabouraud for $24 \mathrm{~h}$ at $35^{\circ} \mathrm{C}$, samples were inoculated in potato agar with fluconazole (8.0 $\mu \mathrm{g} / \mathrm{ml}$ ) and incubated for $48 \mathrm{~h}$. After that, successive transfers were performed for potato agar medium added with fluconazole in the concentrations of $16,32,64,128$ e 256 $\mu \mathrm{g} / \mathrm{ml}$. Colonies were randomly selected on the plates of greater concentrations in which growth could be observed. Subsequently, these colonies were transferred successively to RPMI agar containing increasing concentrations of amphotericin B $(0.5,1.0,1.5,2.0,2.5$ e $3.0 \mu \mathrm{g} / \mathrm{ml})$. Colonies were selected similarly to the procedure of the anterior experiment. These colonies were cultured in Sabouraud agar free of drug at $35^{\circ} \mathrm{C}$ for $24 \mathrm{~h}$ for MIC determination. Induction $B(A B / F z)$ : It was made exactly as induction $\mathrm{A}$, but inverting the antifungal order.

Stability of fluconazole resistance in vitro: Highly resistant sub-clones were transferred daily to Sabouraud agar free of drug and incubated at $35^{\circ} \mathrm{C}$. Minimum inhibitory concentration (MIC) for fluconazole was determined every week for each sub-clone during 30 days using Etest method.
Comparison of original and mutant isolates by length of region ITS2: Genomic DNA was extracted from all original isolates and selected mutants by a method described previously (3). The oligonucleotides ITS3 (5'GCATCGATGAAGAACGCAGC-3') and ITS 4 (5'TCCTCCGCTTATTGATATGC-3`) were used as primers for PCR. Amplifications were elaborated in volumes of $50 \mu 1$ containing $20 \mathrm{ng}$ of genomic DNA, 20 pmols of primer, dNTP $(0.2 \mathrm{mM})$ buffer $(10 \mathrm{mM}$ Tris- $\mathrm{HCl}, 50 \mathrm{mM} \mathrm{KCl}, \mathrm{pH}$ 8), $1.5 \mathrm{mM} \mathrm{MgCl}, 10 \mathrm{mM}$ each dATP, dCTP, dTTP, and dGTP, and $1 \mathrm{U}$ of AmpliTaq DNA polymerase. PCR was developed in Applied Biosystems thermal cycler (model 9700) with an initial denaturing of $95^{\circ} \mathrm{C}$ for 6 min followed by 25 cycles of $60 \mathrm{~s}$ at $95^{\circ} \mathrm{C}$ by $30 \mathrm{~s}, 65^{\circ} \mathrm{C}$ by $30 \mathrm{~s}$ and $72^{\circ} \mathrm{C}$ by $30 \mathrm{~s}$ and then a final cycle of $10 \mathrm{~min}$ at $72^{\circ} \mathrm{C}$. Amplification products were separated by electrophoresis in $1.5 \%$ agarose gels stained with ethidium bromide $1.0 \mu \mathrm{g} / \mathrm{ml}$, in $0,5 \times$ Tris-borate-EDTA buffer for $1,5 \mathrm{~h}$ at $80 \mathrm{~V}$. The gel was observed under UV light and the image was stored using the Kodak digital system EDAS-290.

\section{RESULTS}

In order to identify the method of sub-clone obtaining after the procedures of induction and selection, the samples were named as follows: SFA - Sample selected first from media with fluconazole followed by amphotericin B; SAF Sample selected first from media with amphotericin B followed by fluconazole; IFA - Sample obtained by growth in increasing concentrations of fluconazole followed by growth in increasing concentrations of amphotericin B; IAF Sample obtained by growth in increasing concentrations of amphotericin B followed by growth in increasing concentrations of fluconazole.

\section{Heteroresistance phenotype obtained by selection:}

Selection A: In the screening stage, no sample growth in the presence of amphotericin B $(1.0 \mu \mathrm{g} / \mathrm{ml})$ and three nonalbicans samples did not grow in the presence of fluconazole $(8 \mu \mathrm{g} / \mathrm{ml})$ in the concentration inoculated $\left(10^{4}\right.$ and $10^{7}$ 
$\mathrm{CFU} / \mathrm{ml}$ ). After the selection tests, it was obtained one clone of each sample (425-SP3/06/SFA, 432-SP3/06/SFA e 442SP3/06/SFA), which were able to growth in the presence of fluconazole and, in sequence, amphotericin B.

Selection B: Only seven samples did not demonstrate growth in medium with amphotericin B. $(1.0 \mu \mathrm{g} / \mathrm{ml})$ in $24 \mathrm{~h}$. Samples that showed at least one colony after $48 \mathrm{~h}$ were not used. After the first stage of the selection, beginning with amphotericin B, only the samples 418-SP3/06, 435-SP3/06, 438-SP3/06, 439-SP3/06 e 444-SP3/06 evidenced visible growth in RPMI medium with amphotericin B $(1.0 \mu \mathrm{g} / \mathrm{ml})$. On the second stage, three of those five samples were able to grow in potato agar with fluconazole $(8.0 \mu \mathrm{g} / \mathrm{ml})$. Those samples were considered as having resistance phenotypes and named 418-SP3/06/SAF, 435-SP3/06/SAF, e 438SP3/06/SAF. None of the samples studied presented decrease in MIC values for fluconazole. For amphotericin B, some samples had a slight decrease in MIC values after the selection, as sample 425-SP3/06/SFA that had MIC of $0.25 \mu \mathrm{g} / \mathrm{ml}$ before and $0.12 \mu \mathrm{g} / \mathrm{ml}$ after the procedures (CLSI method). However, this increase was not greater than one dilution of difference.

\section{Heteroresistance phenotypes obtained by induction:}

Induction A: The susceptible samples with greater MICs for fluconazole, 420-SP3/06, 429-SP3/06 e 434-SP3/06, were selected for the induction tests, beginning with fluconazole. They were able to develop growth in all fluconazole (16.0, $32.0,64.0,128$ and $256 \mu \mathrm{g} / \mathrm{ml})$ and amphotericin B $(0.5,1.0$, $1.5,2.0,2.5$ and $3.0 \mu \mathrm{g} / \mathrm{ml}$ ) concentrations, and because of that, were considered resistance phenotypes and named 420SP3/06/IFA, 429-SP3/06/IFA e 434-SP3/06/IFA.

Induction B: At this stage, the wild samples 420-SP3/06, 425-SP3/06 e 428-SP3/06, were selected for the induction evaluation because the MIC values obtained for these samples were higher than the values obtained for the others. The samples were capable to grow in amphotericin B at the concentrations of $2.5,3.0$ e $2.0 \mu \mathrm{g} / \mathrm{ml}$ respectively, and, after that, grew in all concentrations of fluconazole evaluated. Because of these results, the samples were identified as resistance phenotypes and named 420-SP3/06/IAF, 425SP3/06/IAF e 428-SP3/06/IAF. The sample 425-SP3/06/IAF, initially classified as susceptible (MIC $\leq 2.0 \mu \mathrm{g} / \mathrm{ml}$ ), showed MIC of $64.0 \mu \mathrm{g} / \mathrm{ml}$ with both CLSI and Etest methods, being, consequently, resistants. The sample 434-SP3/06/IFA, initially defined as intermediately susceptible to fluconazole $(\mathrm{MIC}<8.0 \mu \mathrm{g} / \mathrm{ml})$, after induction, showed intermediate susceptibility to fluconazole using CLSI methodology $(\mathrm{MIC}=8.0 \mu \mathrm{g} / \mathrm{ml}$ ), being resistant by the Etest methodology $(\mathrm{MIC}=64.0 \mu \mathrm{g} / \mathrm{ml})$. MIC values before and after selection are represented on table 1. Although the sample 428-SP3/06 to be, a priori, resistant to fluconazole, it was employed in the tests of induction in order to obtain samples with cross resistance to amphotericin B. However, no alteration of MIC, to this antifungal after induction, was detected.

\section{In vitro susceptibility test of wild samples and their} resistant clones: Two resistant clones originated from 425SP3/06 isolate were produced by selection on plates with fluconazole $8 \mu \mathrm{g} / \mathrm{ml}$ (425-SP3/06/SFA) as well as by induction on plates with increasing concentration of fluconazole (425-SP3/06/IAF). MICs were evaluated by Etest $(\mathrm{MIC} \geq 48 \mu \mathrm{g} / \mathrm{ml}$ ) and microdilution ( $\mathrm{MIC}=64 \mu \mathrm{g} / \mathrm{ml})$. The samples 16-SAF and 26-SAF, originated by selection, showed a slight increase in MIC values for fluconazole, but kept the classification of sensitive. MICs presented by resistant clones were very greater than those for wild isolates, suggesting heteroresistance.

The variance between MICs verified by broth microdilution and agar-based methods may be attributed to the enhanced growth produced by the increased amount of glucose ( $2 \%$ supplementation) contained in the Mueller Hinton agar medium. Similar medium-specific differences have been observed previously $(3,7)$ (Table 1$)$. 
Table 1. Susceptibility to amphotericin B and fluconazole of Candida spp strains before and after selection and induction of heteroresistance.

\begin{tabular}{|c|c|c|c|c|}
\hline \multirow{3}{*}{$\begin{array}{c}\text { Strains } \\
\text { Selection }\end{array}$} & \multicolumn{4}{|c|}{ Initial MIC / Final MIC ( $\mu \mathrm{g} / \mathrm{ml})$} \\
\hline & \multicolumn{2}{|c|}{ Fluconazole } & \multicolumn{2}{|c|}{ Amphotericin B } \\
\hline & Etest & CLSI & Etest & CLSI \\
\hline $425-\mathrm{SP} 3 / 06 / \mathrm{SFA}^{\mathrm{b}}$ & $0.75 / 48.0$ & $0.12 / 64.0$ & $0.190 / 0.230$ & $0.250 / 0.12$ \\
\hline $432-\mathrm{SP} 3 / 06 / \mathrm{SFA}^{\mathrm{b}}$ & $0.38 / 0.75$ & $0.12 / 0.50$ & $0.064 / 0.640$ & $0.120 / 0.25$ \\
\hline $442-\mathrm{SP} 3 / 06 / \mathrm{SFA}^{\mathrm{b}}$ & $0.50 / 1.00$ & $0.12 / 0.12$ & $0.190 / 0.016$ & $0.250 / 0.25$ \\
\hline $418-\mathrm{SP} 3 / 06 / \mathrm{SAF}^{\mathrm{c}}$ & $0.38 / 0.50$ & $0.12 / 0.25$ & $0.023 / 0.023$ & $0.015 / 0.12$ \\
\hline $435-\mathrm{SP} 3 / 06 / \mathrm{SAF}^{\mathrm{c}}$ & $0.50 / 0.75$ & $0.12 / 0.12$ & $0.094 / 0.047$ & $0.015 / 0.25$ \\
\hline $438-\mathrm{SP} 3 / 06 / \mathrm{SAF}^{\mathrm{c}}$ & $0.75 / 1.00$ & $0.12 / 0.12$ & $0.047 / 0.230$ & $0.015 / 0.25$ \\
\hline \multicolumn{5}{|l|}{ Induction } \\
\hline 420-SP3/06/IFA ${ }^{\mathrm{d}}$ & $0.50 / 0.50$ & $0.12 / 0.25$ & $0.125 / 0.032$ & $0.015 / 0.25$ \\
\hline 429-SP3/06/IFA ${ }^{d}$ & $0.75 / 1.00$ & $0.25 / 0.12$ & $0.047 / 0.047$ & $0.015 / 0.12$ \\
\hline 434-SP3/06/IFA ${ }^{d}$ & $4.00 / 48.0$ & $4.00 / 8.00$ & $0.047 / 0.023$ & $0.015 / 0.12$ \\
\hline 420-SP3/06/IAF ${ }^{\mathrm{e}}$ & $0.50 / 1.00$ & $0.12 / 1.00$ & $0.125 / 0.016$ & $0.015 / 0.12$ \\
\hline $425-\mathrm{SP} 3 / 06 / \mathrm{IAF}^{\mathrm{e}}$ & $0.75 / 64.0$ & $0.12 / 64.0$ & $0.190 / 0.380$ & $0.250 / 0.25$ \\
\hline 428-SP3/06/IAF ${ }^{\mathrm{e}}$ & $48.0 / 64.0$ & $64.0 / 64.0$ & $0.500 / 0.380$ & $0.030 / 0.25$ \\
\hline
\end{tabular}

Stability of fluconazole resistance in vitro: The highly resistant sub-clones of strains 425-SP3/06/SFA, 425SP3/06/IAF and 434-SP3/06/IFA were transferred to drugfree medium to assess the stability of resistance. For the strain 425-SP3/06/SFA, in which resistance was determined by selection, the phenotype was stable. However, for the others two strains, 425-SP3/06/IAF and 434-SP3/06/IFA, the phenotype was reversible after serial transfers in fluconazolefree medium at $35^{\circ} \mathrm{C}$ (Table 2).

Table 2. Evaluation of the stability of the fluconazole resistance phenotype of Candida spp. samples obtained by selection and induction.

\begin{tabular}{ccccc}
\hline Samples & \multicolumn{4}{c}{ MIC in four weeks $(\boldsymbol{\mu g} / \mathbf{m l})$} \\
\cline { 2 - 5 } & First & Second & Third & Fourth \\
\hline 425-SP3/06/SFA & 48 & 32 & 24 & 24 \\
425-SP3/06/IAF & 64 & 16 & 4 & 2 \\
434-SP3/06/IFA & 48 & 16 & 16 & 4 \\
\hline
\end{tabular}




\section{Comparison of original and mutant isolates}

There was no discrepancy between the size of the fragments observed for the mutant strains and their respective wild isolates.

\section{DISCUSSION}

The impact of the use of fluconazole and its correlation with the emergence of Candida spp. resistant strains is a very relevant subject that has been discussed since the 80 's decade. The results presented in the present work evidence individual differences among Candida species. These differences might be important to the choice of the antifungal therapy. However, much more important than the variation between species is the level of protection to the exposure to fluconazole found in C. albicans.

Differences $(0.125$ to $64.0 \mu \mathrm{g} / \mathrm{ml})$ in the susceptibility to fluconazole between the isolate 425-SP3/06 and the other wild samples might be related to the pre-exposure of this sample the antifungal agent before isolation. It has been previously proved that the prophylactic use of fluconazole in invasive fungal infection may lead to the increase in the colonization by yeasts, because samples resistant to fluconazole might be selected with this exposure $(11,19)$. Indeed, according to Vasques et al. (27), samples less susceptible to fluconazole can be isolated from patients in therapy with this antifungal agent.

The first article focused on the heteroresistance phenomenon was published in 1997, reporting the studies elaborated by Schoofs et al. (24). These authors observed that, when growth was performed in the presence of azole compounds, samples obtained from the oral cavity of AIDS patients and isolated in medium containing antifungal showed faster growth kinetics than those ones obtained in medium without drug. This fact suggest that the initial isolation of colonies in medium containing drug had selected sub-populations with greater resistance to azoles, since this colonies showed growth within the expected pattern, even in the presence of the antifungal. The same fact was not observed with colonies isolated in medium without drug.
According to reports found in the literature, the occurrence of heteroresistance in Candida is frequent and might be caused by two basic mechanisms: a) Reversible increased expression of efflux pumps $(4,7,23)$. In this case, there is a direct correlation between the drug exposure and the maintenance of the phenomenon (4); b) Irreversible heteroresistance related to the alteration in the sterol content and alteration in the cellular targets for the antifungal agents $(4,7)$.

The results of this work demonstrate the occurrence of heteroresistance to fluconazole in two $C$. tropicalis strains (425-SP3/06/SFA and 425-SP3/06/IAF) and one C. glabrata (434-SP3/06/IFA). These samples were produced by different methodologies from wild strains 425-SP3/06 and 434SP3/06, respectively. The other samples denoted small differences in MIC values, remaining susceptible to the drug. Furthermore, it has been demonstrated that the development of resistance due to the use of antifungal drugs is much more frequent among other Candida species than in C. albicans (2, 28). The evaluation of clinical isolates indicates that resistance is not exclusively due to $C$. albicans resistant isolates but also to the increase in the number of non-albicans Candida isolates (14).

The procedures employed aiming the selection of antifungal resistant clones are particularly important in the evaluation of the potential of Candida strains in order to develop other mechanisms to escape from the antifungal action. This occurs because the levels of the drug in blood might suppress the growth of susceptible yeasts, but still allow the growth of resistant strains. In this way, some of these microorganisms might quickly develop resistance or need higher drug dosages and can be responsible for fungal infections in susceptible individuals.

Although the prophylactic use of fluconazole is an important factor to the appearance of resistance, there are other determinant aspects involved in the selection of nonalbicans Candida species. In fact, basis diseases associated with immunosuppression and exposure to invasive medical procedures have been cited as relevant factors for the 
occurrence of infection caused by non-albicans Candida species, including C. glabrata (10). It has been described that infections caused by this yeast might occur in patients previously exposed to amphotericin B $(10,17)$.

In this work, only one sample (3.33\%) with stable resistance phenotype was produced after successive transfers for free of drug medium through the methodology for selection of heteroresistant strains presently used. It is possible that this unique sample already existed in the initial colonies, being selected from a sub-population with some kind of irreversible mutation, which would lead to resistance to fluconazole. There are different types of biochemical mechanisms that contribute to the heteroresistance phenotype, which would explain the results presented in this paper. Among these mechanisms, it can be mentioned the alteration in the sterol content and changes in the cellular antifungal targets, that remained stable after successive transfers $(13,23)$. Considering the molecular point of view, biochemical alterations might result from genetic amplification, transference or deletion, point mutations, transcriptional activation and hypo or hyper-metylation. All these effects might occur in genes involved on the regulation or in the process of the biochemical pathway $(7,25)$.

None of the species analyzed in this work showed increase in MIC values for amphotericin B, even after cultivation in media with increasing concentrations of anphotericin B $(0.5$ a $3.0 \mu \mathrm{g} / \mathrm{ml})$. However, cells that have adaptations to this antifungal agent are not classified as resistant by the standard in vitro methods after subcultivation in media without the drug. These results are in agreement with the data reported by Vazques et al. (27), which shows that $C$. albicans, the most sensitive to amphotericin B species, is able to growth in high concentrations of this polyene after pre-exposure to azoles. These authors suggest that the sequential treatment with fluconazole followed by amphotericin B might represent a risk of therapeutic failure, due to the ability of Candida species to grow in vitro in the presence of amphotericin B after pre-exposure to fluconazole. Besides, even in simultaneous therapies, it is possible to detect the effects in vitro, since in the patient there are pharmacokinetic differences between the two drugs. On the other hand, data published by Pfaller et al. (20), regarding C. glabrata strains with acquired resistance to fluconazole, demonstrated that this pathogen has, more frequently, high MIC values for amphotericin B.

The sample 425-SP3/06/IAF (C. tropicalis), heteroresistant to fluconazole, originated from the experimental induction with amphotericin B and fluconazole, showed high MIC values $(\mathrm{MIC}=64 \mu \mathrm{g} / \mathrm{ml})$ in all methods of susceptibility determination employed. However, after successive transfers in medium free of drug, the sample showed reversion of the resistance phenotype. Barchiesi et al. (1), also focusing C. tropicalis, used a methodology similar to the procedure employed in this paper for the obtaining of strains heteroresistant to fluconazole, itraconazole and amphotericin B. They verified that, as it occurred with 425-SP3/06/IAF, all the heteroresistant samples originated by induction, showed reversion of the phenotype. Several studies have shown that the instability of these phenotypes is correlated with the expression of the efflux pumps $(5,9,13)$. These mechanisms might explain the instability of the heteroresistance phenotype acquired for both 425-SP3/06/IAF and 434-SP3/06/IFA samples. However, Goldman et al. (8) established that there is no linear correlation between MIC values and the increase in the expression of the genes that codify efflux pumps. Thus, molecular approaches for the gene expression of the mutant samples and their respective wild samples are necessary to elucidate this question.

Although some clones of the initial isolates have grown in the medium containing antifungal in the screening stage, the MIC values were relatively low, conserving the yeasts with their susceptibility phenotype. Xu et al. (29), working with Cryptococcus neoformans, have also found isolates that, after the screening stage, showed low MIC values for fluconazole $(0.5$ and $1.0 \mu \mathrm{g} / \mathrm{ml})$. These results mentioned above could be explained by the ability of some strains in 
produce physiologic transitory adaptations or transitory tolerance to fluconazole.

Considering that mutant strains and their respective wild samples showed the same band profile in the PCR of the ITS2 region, it is possible to conclude that any contamination occurred.

Turenne et al. (26) demonstrated that C. albicans, $C$. tropicalis, C. parapsilosis, C. glabrata, and other fungal species showed, individually, distinct sizes of ITS2 fragments. Moreover, other authors verified that the region ITS1 also express inter-species variability based in the size of the fragments (12).

The widespread and inadequate use of antifungal drugs may explain the inefficacy of the treatments of candidemias. One of the main factors for the failure of these therapies might be the increase of the resistance in Candida spp. to antifungal agents. This resistance occurs by a process of natural induction or selection. Infection with Candida susceptible to the drug has been gradually substituted by infections caused by more resistant species. Among the most susceptible are C. albicans and among the less susceptible are C. glabrata and C. krusei. Failures in treatments are leading the scientists to search for drugs with other targets of action.

The data of the present article established that strains heteroresistant to fluconazole may be originated from previous exposure to fluconazole. Mondon et al. (15) investigated the occurrence of resistance to fluconazole and voriconazole in patients with or without pre-exposure to drugs and suggested that resistance seems an innate phenomenon. Goldman et al. (8) observed that the mechanisms of resistance, including point mutations in the ERG11 gene and over-expression of this gene or others that codify efflux pumps, may be found even in susceptible isolates and, consequently, the heteroresistance phenotype is not related to these isolated mechanisms.

The resistance to fluconazole documented in this study suggests that the exposure to this drug leads to the occurrence of colonization or infection by resistant populations, selected or induced during the treatment.
The mechanisms involved in the resistance to fluconazole in the samples 425-SP3/06/SFA and 425SP3/06/IAF should be elucidated and compared by molecular analyses, because these samples were obtained from the same wild isolate, susceptible to the drug. These studies may contribute for the better comprehension of the mechanisms involved in the resistance of Candida species to fluconazole.

\section{REFERENCES}

1. Barchiesi, F.; Calabrese, D.; Sanglard, D.; Caselli, F.; Giannini, D.; Giacometti, A.; Gavaudan, S.; Scalise, G. (2000). Experimental induction of fluconazole resistance in Candida tropicalis ATCC 750. Antimicrob. Agents Chemother. 44, 1578-1584.

2. Bodey, G.P. (2001). Azole antifungal agents. Clin Infect Dis. 14, 5161 5169.

3. Bolano, A.; Stinchi, S.; Preziosi, R.; Bistoni, F.; Allegrucci, M.; Baldelli, F.; Martini. A.; Cardinali, G. (2001). Rapid methods to extract DNA and RNA from Cryptococcus neoformans. FEMS Yeast Res. 1, 221-224.

4. Bouchara, J.P.; Zouhair, R.; Le Boudouil, S.; Renier, G.; Filmon, R.; Chabasse, D.; Hallet, J.N.; Defontaine, A. (2000). In-vivo selection of an azole-resistant petite mutant of Candida glabrata. J. Med. Microbiol. 49, 977-984.

5. Cowen, L.E.; Sanglard, D.; Calabrese, D., Sirjusingh C, Anderson JB Kohn LM (2000). Evolution of drug resistance en experimental populations of Candida albicans. J. Bacteriol. 3, 1515-1522.

6. Cuenca-estrella, M.A.; Gomez-Lopez, A.; Mellado, E.; RodriguezTudela, J.L. (2005). Correlation between the procedure for antifungal susceptibility testing for Candida spp. of the European Committee on Antibiotic Susceptibility Testing (EUCAST) and four commercial techniques. Clin. Microbiol. Infect. 11, 486-492.

7. Defontaine, A.; Bouchara, J.P.; Declerk, P.; Planchenault, C.; Chabasse, D.; Hallet, J.N. (1999). In-vitro resistance to azoles associated with mitochondrial DNA deficiency in Candida glabrata. J. Med. Microbiol. 48, 663-70.

8. Goldman, G.H.; da Silva Ferreira, M.E.; dos Reis Marques, E.; Savoldi, M.; Perlin, D.; Park, S.; Godoy Martinez, P.C.; Goldman, M.H.; Colombo, A.L. (2004). Evaluation of fluconazole resistance mechanisms in Candida albicans clinical isolates from HIV-infected patients in Brazil. Diagn. Microbiol. Infect. Dis. 50, 25-32.

9. Karl, W.H.; Joseph, T.N.; Thomas, D.E. (2000). Upregulation of ERG genes in candida species by azoles and other sterol biosynthesis inhibitors. Antimicrobial Agents Chemother. 44, 2693-2700.

10. Krcmery, V.; Barnest, A.J. (2002). Non-albicans Candida spp. Causing fungaemia: pathogenicity and antifumgal resistance. J. Hosp. Infect. 50, 243-260. 
11. Laverdiere, M.; Rotstein, C.; Bow, E.J.; Roberts, R.S.; Ioannou, S.; Carr, D.; Moghaddam, N. (2000). Impact of fluconazole prophylaxis on fungal colonization and infection rates in neutropenic patients. $J$. Antimicrob. Chemother. 46, 1001-1008.

12. Lott, T.J.; Kuykendall, R.J.; Reiss, E. (1993). Nucleotide sequence analysis of the 5.8S rDNA and adjacent ITS2 region of Candida albicans and related species. Yeasts 9, 1199-1206.

13. Marichal. P.; Koymans, L.; Willemsens, S.; Bellens, D.; Verhasselt, P.; Luyten, W.; Borgers, M.; Ramaekers, F.C.S.; Odds, F.C.; Vanden Bossche, H. (1999). Contribuition of mutations in the cytochrome P450 $14 \alpha$-demethylase (ERG11p, Cyp51p) to azole resistence in Candida albicans. Microbiol. 145, 2701-2713.

14. Moran, G.P.; Sullivan, D.J.; Henman, M.C.; McCreary, C.E.; Harrington, B.J.; Shanley, D.B.; Coleman, D.C. (1997). Antifungal drug susceptibilities of oral Candida dubliniensis isolates from human immunodeficiency virus (HIV)-infected and nonHIV-infected subjects and generation of stable fluconazole-resistant derivatives in vitro. Antimicrob. Agents Chemother, 41. 617-623.

15. Mondon, P.; Petter, R.; Amalfitano, G.; Luzzati, R.; Concia, E.; Polacheck, I.; Kwon-Chung, K.J. (1999). Heteroresistance to fluconazole and voriconazole in Cryptococcus neoformans. Antimicrob. Agents Chemother. 43, 1856-1861.

16. Clinical and Laboratory Standards Institute (2002) Reference method for broth dilution antifungal susceptibility testing of yeasts. Approved standard (NCCLS document M27-A2) Villanova, PA.

17. Nucci, M.; Colombo, A.L. (2002). Emergence of resistant Candida in neutropenic patients. Braz. J. Infect. Dis. 6, 124-128.

18. Nunes, A.P.; Schuenck, R.P.; Bastos, C.C.; Magnanini, M.M.; Long, J.B.; Iorio, N.L.; Santos, K.R. (2007). Heterogeneous resistance to vancomycin and teicoplanin among Staphylococcus spp. isolated from bacteremia. Braz. J. Infect. Dis. 3, 345-350.

19. Petrikkos, G.; Skiada, A. (2007). Recent advances in antifungal chemotherapy. Int. Antimicrob. Agents. 1, 108-117.

20. Pfaller, M.A.; Messer, S.A.; Hollis, R.J.; Jones, R.N.; Diekema, D.J. (2002). In vitro activities of ravuconazole and voriconazole compared with those of four approved systemic antifungal agents against 6,970 clinical isolates of Candida spp. Antimicrobial Agents Chemother. 46, 1723-1727.

21. Pfaller, M.A.; Boyken, L.; Messer, S.A.; Tendolkar, S.; Hollis, R.J.; Diekema, D.J. (2004). Evaluation of the Etest Method Using MuellerHinton Agar with Glucose and Methylene Blue for Determining Amphotericin B MICs for 4,936 Clinical Isolates of Candida Species. J. Clin. Microbiol. 42, 4977-4979.

22. Ruhnke, M. (2006). Epidemiology of Candida albicans infections and role of non-Candida-albicans yeasts. Curr. Drug Targets. 7, 495-504.

23. Sanglard, D. (2002). Clinical relevance of mechanisms of antifungal drug resistance in yeasts. Enferm. Infec. Microbial. Clin. 20, 462-470.

24. Schoofs, A.; Odds, F.C.; Colebunders, R.; Ieven, M.; Wouters, L.; Goossens, H. (1997). Isolation of Candida species on media with and without added fluconazole reveals high variability in relative growth susceptibility phenotypes. Antimicrob. Agents Chemother. 41, $1625-$ 1635

25. Silva, V.M.; Cristina Díaz, J.; Naldy Febré, Y.; Red, D.E .(2002) Diagnóstico en micología médica.Vigilancia de la resistencia de leveduras a antifúngicos. Rev. Chil. Infectol. Santiago 19.

26. Turenne, C.Y.; Sanche, S.E.; Hoban, D.J.; Karlowsky, J.A.; Kabani, A.M. (1999). Rapid Identification of Fungi by Using the ITS2 Genetic Region and an Automated Fluorescent Capillary Electrophoresis System. J. Clin. Microbiol. 37, 1846-1851.

27. Vazques, J.A.; Arganoza, M.T.; Boikov, D.; Yoon, S.; Sobel, J.D.; Akins, R.A. (1998). Stable phenotypic resistance of Candida species to amphotericin B conferred by preexposure to subinhibitory levels of azoles. J. Clin. Microbiol. 36, 2690-2695.

28. Walsh, T.J.; Pizzo, A. (1988). Treatment of systemic fungal infections: recent progress and current problems. Eur. J. Clin. Microbiol. Infect. Dis. 7, 460-475.

29. Xu, J.; Onyewu, C.; Yoell, H.J.; Ali, R.Y.; Vilgalys, R.J.; Mitchell, T.G. (2001). Dynamic and heterogeneous mutations to fluconazole resistance in Cryptococcus neoformans. Antimicrob. Agents Chemother. $45,420-427$. 